\title{
Estimulación prenatal en gestantes de alto riesgo obstétrico: una mirada desde la ocupación
}

Temática: práctica basada en la evidencia, promoción y prevención.

Aporte a la disciplina: la terapia ocupacional es una alternativa de apoyo especializado a las mujeres embarazadas de alto riesgo. Reconoce al feto como un ser capaz de actuar y reaccionar en el vientre materno frente a estímulos intrauterinos de tipo visual, táctil, motor y auditivo, y motiva la sensibilidad e interacción de la madre frente a su hijo por nacer. El trabajo de los terapeutas ocupacionales se dirige al modelado de las características de las gestantes, al rendimiento ocupacional y a la creación de adaptaciones y modificaciones en las actividades y ambientes ocupacionales. Utilizan estrategias de enseñanza-aprendizaje que fomentan nuevos modos de conocer, formas expresivas y creativas que pueden trasformar las prácticas de estimulación prenatal; facilitan la realización de actividades que permitan a las gestantes incorporar la capacidad, eficacia y goce en los procesos adquiridos y realizados en las ocupaciones productivas y significativas y en la participación en ocupaciones con significado y propósito.

\section{RESUMEN}

Objetivo: reeducar gestantes de alto riesgo obstétrico en prácticas de estimulación prenatal y ocuparlas en actividades que mejoren su calidad de vida mientras están hospitalizadas. Materiales y métodos: estudio cualitativo de investigación acción participativa durante el segundo semestre de 2015 y el primer semestre de 2016, mediante caracterización sociodemográfica, grupos focales de conocimiento de experiencias y prácticas de las madres, negociación de talleres educativos de estimulación prenatal, ejecución de actividades ocupacionales y evaluación de cambios. Resultados: participaron 35 gestantes entre 18 y 41 años, de las cuales el $80 \%$ fueron diagnosticadas con preeclampsia, y el $50 \%$ tenían menos de 33 semanas gestacionales. Se encontraron diferencias y similitudes en los conocimientos y las prácticas sobre estimulación prenatal, así como la necesidad de mejorarlos. La negociación de los talleres educativos propició la realización e incorporación de prácticas como actividad ocupacional con significado; la elaboración de un juguete para

\section{DOI: 10.5294/aqui.2018.18.4.5}

Para citar este artigo / Para citar este artículo / To reference this article

Rubio-Grillo MH, Perdomo-Oliver NM, Zamudio-Espinosa DC. Estimulación prenatal en gestantes de alto riesgo obstetrico, una mirada desde la ocupación. Aquichan 2018; 18(4): 426-437. DOI: 10.5294/aqui.2018.18.4.5

$1 \bowtie$ orcid.org/0000-0002-3612-7885. Escuela de Rehabilitación Humana, Universidad del Valle, Colombia. maria.rubio@correounivalle.edu.co

2 orcid.org/0000-0003-0247-9687. Escuela de Rehabilitación Humana, Universidad del Valle, Colombia. nilia.perdomo@correounivalle.edu.co

Recibiddo: 16/08/2018 Enviado a pares: 14/09/2018 Aceptado por pares: 10/10/2018 Aprobado: 26/10/2018 
el bebé generó curiosidad, exploración, habilidades o formas ocupacionales y desempeño consistente en respuesta a demandas del ambiente. Conclusiones: al realizar actividades con sentido y propósito, las gestantes incorporaron la capacidad, eficacia y goce en los procesos adquiridos y realizados. Estar ocupadas les permitió implementar nuevas formas de saber y hacer, así como motivarse por encontrar actividades significativas en el reaprendizaje de la estimulación prenatal.

\section{PALABRAS CLAVE (FUENTE: DECS)}

Mujeres embarazadas; relaciones materno-fetales; educación prenatal; terapia ocupacional; terapia con arte.

\section{Prenatal Stimulation in Pregnant Women with High Obstetric Risk: A Perspective from Activity}

\section{ABSTRACT}

Objectives: To reeducate pregnant women with high obstetric risk in prenatal stimulation practices and to involve them in activities that improve their quality of life while hospitalized. Materials and methods: Qualitative study with a participatory action research approach conducted during the second half of 2015 and the first half of 2016, through sociodemographic characterization, focus groups of knowledge of mothers' experiences and practices, negotiation of educational prenatal stimulation workshops, performance of occupational activities, and evaluation of changes. Results: Thirty-five pregnant women between 18 and 41 years old participated. $80 \%$ were diagnosed with preeclampsia and $50 \%$ were less than 33 weeks pregnant. Differences and similarities were found in prenatal stimulation knowledge and practices, as well as the need to improve them. Negotiating educational workshops promoted the use and incorporation of practices as meaningful occupational activities; making a toy for the baby caused curiosity, exploration, occupational skills and consistent performance in response to environmental demands. Conclusions: In carrying out activities with sense and purpose, pregnant women incorporated ability, effectiveness and enjoyment in the processes conducted. Being busy allowed them to implement new ways of knowing and doing and motivated them to find meaningful activities in relearning prenatal stimulation.

\section{KEYWORDS (SOURCE: DeCS)}

Pregnant women; maternal-fetal relations; prenatal education; occupational therapy; art therapy. 


\section{Estimulação pré-natal em manipuladores obstétricos de alto rïsco: um ollhar da ocupação}

\section{RESUMO}

Objetivo: reeducar gestantes de alto risco obstétrico nas práticas de estimulação pré-natal e realizar atividades que melhorem a qualidade de vida durante a internação. Materiais e métodos: estudo qualitativo da Pesquisa Ação Participativa, durante o segundo semestre de 2015 e o primeiro semestre de 2016. Por meio de caracterização sóciodemográfica, grupos focais de conhecimento de experiências e práticas de mães, negociação de oficinas educativas de estimulação pré-natal, execução de atividades ocupacionais e avaliação de mudanças. Resultados: participaram 35 gestantes, entre 18 e 41 anos, das quais $80 \%$ foram diagnosticadas com pré-eclâmpsia e $50 \%$ tinham menos de 33 semanas de gestação. Além disso, diferenças e semelhanças foram encontradas no conhecimento e nas práticas de estimulação pré-natal, bem como na necessidade de aprimorá-las. A negociação de oficinas educativas levou à realização e incorporação de práticas como: atividade ocupacional com significado; a elaboração de um brinquedo para o bebê; exploração, habilidades e/ou formas ocupacionais e desempenho consistente em resposta às demandas ambientais. Discussão e conclusão: ao realizar atividades com significado e propósito, as gestantes incorporaram a capacidade, a eficácia e o prazer nos processos adquiridos e realizados. 0 fato de estarem ocupadas permitiu que elas implementassem novas formas de conhecer e fazer, bem como que estivessem motivadas para encontrar atividades significativas na reaprendizagem da estimulação pré-natal.

PALAVRAS-CHAVE (FONTE: DECS)

Mulheres grávidas; relações materno-fetais; educação pré-natal; terapia ocupacional; terapia com arte. 


\section{Introducción}

El embarazo de alto riesgo incrementa la probabilidad de enfermar o morir antes, durante o después del parto (1) y constituye un problema de salud pública mundial, con amplias brechas entre países según su desarrollo económico. En el mundo mueren 800 mujeres por causas relacionadas con el embarazo y el parto, de las cuales el $99 \%$ vive en países en desarrollo (2). En Latinoamérica, la cifra es de 80 por cada 100.000 nacidos vivos, y se observan diferencias entre los países norteamericanos y los latinoamericanos: mientras Canadá presenta 12 casos, Colombia, 71,64, y Haití, 325 por cada 100.000 nacidos vivos (3). En el Valle del Cauca (4), la tasa de mortalidad neonatal es de 7,24 casos por 1000 habitantes, es decir, más baja que la nacional.

La Organización Mundial de la Salud recomienda mejorar la calidad de la atención prenatal, reducir el riesgo de muertes prenatales y complicaciones del embarazo y brindar una experiencia positiva durante la gestación (5). Es necesario desarrollar un programa educativo, tanto para la madre, como para la familia, así como favorecer los factores personales y ambientales que la protegen, ampliar su seguridad, mejorar su nivel de satisfacción frente a los servicios prestados, ejercerles un mejor control de bienestar físico, mental y social, y contar con un apoyo especializado para las madres gestantes en alto riesgo $(6,7)$.

La estimulación prenatal durante la gestación motiva y promueve el vínculo afectivo madre-hijo. Es parte de la preparación psicoafectiva de la psicoprofilaxis obstétrica (8), implica estímulos intrauterinos visuales, táctiles, motores y auditivos (9), y es una estrategia de promoción de la salud materna y neonatal, dependiente de la interacción entre factores biológicos y psicosociales $(8,10)$. Una oportuna y acertada estimulación enriquece y optimiza el desarrollo físico y mental del ser intrauterino, porque permite establecer la mayor cantidad de sinapsis neuronales y capacidad de comunicaciones en la masa cerebral $(11,12)$.

La estimulación prenatal la ejecuta un amplio grupo de profesionales de la salud especializados, como auxiliares de enfermería, fisioterapeutas, terapeutas ocupacionales, enfermeros, matronas y médicos $(12,13)$, todos pensando y actuando desde donde cada uno puede aportar. En el contexto hospitalario (14), las madres pueden educarse en el manejo prenatal para prevenir factores de riesgo en el desarrollo del bebé (15), así como participar en intervenciones de terapia ocupacional que complementen la estimulación prenatal (16).
En Latinoamérica, se destacan por sus programas de estimulación prenatal Cuba (17) y Perú (9), y en el país, Sincelejo (18) y Tunja (19). Su abordaje de la estimulación prenatal no aplica un proceso de aprendizaje-acción enfocado en la ocupación con significado, sino es limitado; por lo tanto, es relevante analizar y concertar, a partir de los conocimientos, percepciones y prácticas, alternativas sobre la estimulación prenatal.

Así, el propósito de esta investigación fue analizar los conocimientos, experiencias y percepciones sobre la estimulación prenatal de las gestantes hospitalizadas en una unidad de alto riesgo obstétrico (ARO) en un hospital de tercer nivel de la ciudad de Cali, concertar actividades creativas con sentido y propósito y reeducarlas en la aplicación de la estimulación prenatal.

\section{Materiales y métodos}

Se realizó una investigación acción participativa (IAP) (20), correspondiente al paradigma crítico dialéctico con enfoque cualitativo. El diseño seleccionado concibe a las gestantes hospitalizadas como un grupo de personas en alto riesgo, que durante el tiempo libre de su hospitalización no se ocupan de alguna actividad y necesitan reaprender técnicas de estimulación prenatal. Mediante este método, es posible lograr modificaciones en el ámbito hospitalario en cuanto a la participación de gestantes y profesionales de la salud orientados a validar y refinar la estimulación prenatal. Asimismo, es importante la contribución en el proceso de la investigación y la solución del problema, independientemente de los factores personales, sociales y culturales $(20,21)$.

La población estuvo constituida por gestantes hospitalizadas de la Unidad de Alto Riesgo Obstétrico (ARO), en una institución de salud nivel III de Cali, entre el segundo semestre de 2015 y el primero de 2016. No se realizó muestreo; se desarrolló con todas las gestantes que cumplieron con los criterios de inclusión: 1) estancia en la sala de hospitalización durante los periodos de recolección de información; 2) encontrarse entre las 28 y las 40 semanas de gestación; 3) firmar el consentimiento informado manifestando la aceptación para participar en el estudio. Por criterios de exclusión, se descartaron dos madres con contraindicación médica y una menor de 18 años.

Según la Resolución 8430 de 1993 (22), el estudio tuvo una clasificación de "riesgo mínimo" para las gestantes. Contó con el aval del Comité Institucional de Revisión de Ética Humana (CIREH) de las instituciones participantes, con acta de aprobación 
número 010-012 y con el financiamiento por convocatoria interna de la Universidad del Valle, con código interno 1781.

De manera previa al inicio del estudio, se determinaron las necesidades de dimensionar la importancia de la estimulación prenatal en el ámbito hospitalario y se socializaron los objetivos y las razones para ejecutar el proyecto con los directivos, el personal de salud de la sala y las gestantes. La investigación fue llevada a cabo en tres fases: diagnóstico participativo, implementación de estrategias y reflexión; en todas las fases se tuvo un acercamiento cara a cara con las participantes y se efectuó en la sala de hospitalización de la ARO.

En la primera fase se realizó la caracterización sociodemográfica de la población, a partir de la revisión de las historias clínicas. Se llevaron a cabo observaciones no participantes, para obtener una aproximación inicial de las prácticas sobre estimulación prenatal de las madres; se utilizó una matriz de observación de la comunicación entre los padres y el bebé, estimulación táctil, visual, auditiva y motora, orientada por los objetivos del estudio. La captura de datos la realizaron dos monitores seleccionados por el comité de investigación de la Universidad, y entrenados por el equipo de investigadores, a partir de un pilotaje con ajuste de los instrumentos, y supervisión de campo por parte de la investigadora principal. Se consignó la información en un archivo de Excel ${ }^{\circledR}$, y el análisis de la información cuantitativa, en el software StataV $11^{\circledR}$, con la obtención de frecuencias absolutas y relativas para variables cualitativas.

Posteriormente, en un salón que contenía equipos de sonido, grabadora y video, dos de las autoras desarrollaron seis grupos focales. El proceso de cada uno incluyó apertura, preguntas sobre el objeto de estudio y cierre (23). El investigador, como moderador, dirigió la sesión a partir de una guía de indagación sobre los conocimientos, las experiencias y las percepciones de las gestantes sobre la estimulación prenatal; dos estudiantes tomaron nota, realizaron un registro de audio de la sesión, y finalmente se llegó a un consenso colectivo sobre las conclusiones; se agradeció la participación de las madres y se explicó que los datos serían utilizados en la preparación de los talleres. La duración promedio fue de 27 minutos, y se identificó el desconocimiento, prácticas tradicionales, mitos y prejuicios sobre el tema. Participaron las gestantes, los investigadores y una auxiliar de enfermería, para atender cualquier complicación que se presentara.

El análisis de la información de los grupos lo realizaron dos investigadoras y una magíster en salud pública, en el software EthnographV6 $6^{\circledR}$, mediante la codificación axial, con asignación de identificaciones alfanuméricas para cada grupo focal. Durante el análisis surgieron dos categorías emergentes relacionadas con conocimientos generales de estimulación prenatal y coherencia del estado emocional entre la madre y el bebé. Las categorías se organizaron en un árbol de códigos (figura 1).

Figura 1. Árbol de códigos final de la investigación

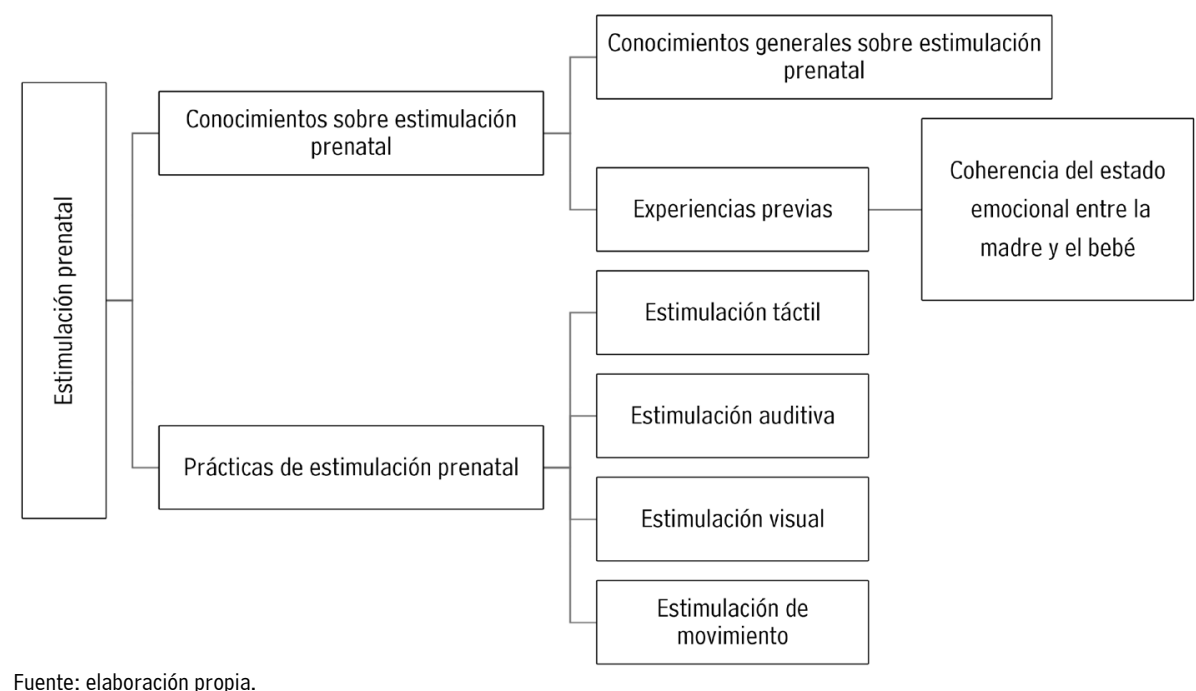

Fuente: elaboración propia. 
La información obtenida se analizó desde un enfoque descriptivo. Se finalizó con la devolución de los resultados parciales a las gestantes, bajo los criterios de rigor de las investigaciones cualitativas: dependencia, credibilidad, transferencia y confirmación (24), con lo cual se dio paso a la siguiente fase.

En la segunda fase se dio a conocer la información obtenida $y$, de forma participativa, se diseñaron e implementaron estrategias, a partir de talleres sobre la estimulación prenatal y actividades terapéuticas con las madres para aminorar el riesgo prenatal y, así, resolver el problema identificado de desconocimiento sobre la estimulación en esta etapa. Dichos talleres se planificaron bajo la orientación de profesionales de terapia ocupacional, y según las necesidades definidas por las gestantes, el equipo de investigadores los efectuó con la participación permanente de las madres.

Las estrategias de estimulación se planearon y ejecutaron según las propuestas de Ludington (25), quien coincide con los roles dominantes que ocupan las madres. Las actividades relacionadas con la estimulación sensorial estuvieron acordes al planteamiento de Ludington (25) y de Verny $(26,27)$, y a la condición de la madre: la estimulación visual consistió en la sensibilización de la diferencia entre el día y la noche; la estimulación táctil se direccionó hacia la aplicación de texturas (de suaves a burdas), con presión de (suave a mediana) de afuera hacia adentro, en movimientos circulares; en cuanto a la estimulación propioceptivo-vestibular, se utilizó el movimiento anteroposterior en posición sedente, de pie, con y sin desplazamiento. Las estrategias aplicadas para la estimulación prenatal están descritas en la (tabla 1).

Según los planteamientos de Aguilar (17), Prieto (19), Bello (28), Lobato (29), Federico (30) y Begoña (31), y la asesoría del ingeniero mecánico-músico, se seleccionó la música de acuerdo con las características de tonalidad, ritmo e instrumentos que cumplieran con los estándares de la estimulación: para el primero, música suave y relajante, que inspirara sentimientos de amor; para el segundo y tercer trimestre, música que facilitara las acciones ocupacionales. Los coordinadores del servicio donde se aplicó la intervención y el coordinador de derechos digitales de SAYCO avalaron la utilización de la música en este tipo de intervención.

La tercera fase incluyó la finalización de los talleres de enseñanza de la estimulación prenatal y la elaboración de un juguete con material desechable, para estimular al bebé una vez naciera. Por medio de una encuesta de satisfacción, se evaluaron las estrategias utilizadas con el fin de indagar sobre la transformación en la implementación de la estimulación prenatal.

Tabla 1. Estímulos, estrategias de estimulación y pistas de audio empleadas en los talleres de estimulación prenatal a madres gestantes hospitalizadas en la Unidad de Alto Riesgo Obstétrico de un hospital nivel III en Cali, 2016

\begin{tabular}{|c|c|c|}
\hline Estímulo & Estrategia de estimulación & Pistas de audio \\
\hline \multirow{2}{*}{ Táctil } & \multirow{2}{*}{ Esponja húmeda con agua } & Meditación (Thaïs), de Jules Massenet [violín y orquesta] \\
\hline & & Canción de la luna (Rusalka), de A. Dvorak [violín y orquesta] \\
\hline \multirow{3}{*}{ Propioceptivo vestibular } & \multirow{3}{*}{ Vaivén } & Air on G String, de J. S. Bach [marimba y flauta] \\
\hline & & Canción de la luna (Rusalka), de A. Dvorak [violín y orquesta] \\
\hline & & Canon in D, de J. Pachelbel [cuerdas y órgano] \\
\hline \multirow[t]{2}{*}{ Visual } & \multirow[t]{2}{*}{ Simulación día-noche } & $\begin{array}{l}\text { Concierto para piano } \text { n. }^{0} \text { 17, } 3 \text { er movimiento, de W. A. Mozart [piano y }_{\text {orquesta] }}\end{array}$ \\
\hline & & Procesión de los nobles, de N. Rimsky-Korsakov [orquesta] \\
\hline
\end{tabular}

Fuente: elaboración propia a partir de los referentes teóricos expuestos. 


\section{Resultados}

\section{Primera fase: diagnóstico participativo}

En la primera fase (caracterización sociodemográfica de las madres y sus condiciones obstétricas) participaron 35 madres gestantes con edades entre 18 y 41 años. La mitad de ellas tenía menos de 33 semanas gestacionales, y el diagnóstico más prevalente fue la preeclampsia no severa (50\%), seguido por la preeclampsia severa (30\%) (tabla 2).

Tabla 2. Datos sociodemográficos de las madres gestantes participantes hospitalizadas en una Unidad de Alto Riesgo Obstétrico de un hospital nivel III (Cali, 2015)

\begin{tabular}{|c|c|c|}
\hline Variable & $\begin{array}{c}\text { Frecuencia } \\
\text { absoluta } \\
(\mathbf{n = 3 5 )}\end{array}$ & $\begin{array}{c}\text { Frecuencia } \\
\text { relativa (\%) }\end{array}$ \\
\hline $\begin{array}{c}\text { Edad gestante en años } \\
\text { cumplidos (Me, RIC) }\end{array}$ & $(25,22-36)$ \\
\hline \multicolumn{3}{|c|}{ Estrato socioeconómico } \\
\hline 1 & 9 & 25,71 \\
\hline 2 & 26 & 74,29 \\
\hline \multicolumn{2}{|c|}{ Escolaridad (n=11) } \\
\hline Técnico & 4 & 36,36 \\
\hline Bachillerato completo & 2 & 18,18 \\
\hline Bachillerato incompleto & 5 & 45,45 \\
\hline $\begin{array}{c}\text { Semanas de gestación } \\
\text { (Me, RIC) }\end{array}$ & $(33,24-36)$ \\
\hline $\begin{array}{c}\text { Días de hospitalización } \\
\text { (Me, RIC) }\end{array}$ & $(9,5,4-14)$ \\
\hline
\end{tabular}

Fuente: elaboración propia.

Las observaciones no participantes realizadas al inicio mostraron que los padres se comunicaron con los profesionales de la salud, y entre ellos en una menor cantidad de ocasiones; la mayoría de las interacciones se realizaron mediante caricias y conversaciones con el bebé en gestación. Por otro lado, la estimulación más practicada fue la visual, con iluminación natural, auditiva mediante el habla, y táctil con aplicación a través del vientre materno. No se apreció la aplicación de técnicas como el ejercicio, la respiración, el equilibrio u otras que permitieran la estimulación motora del feto (tabla 3).
Tabla 3. Principales hallazgos de observaciones no participantes realizadas a madres gestantes hospitalizadas en la Unidad de Alto Riesgo Obstétrico ARO de un hospital nivel III (Cali, 2015)

\begin{tabular}{|c|c|c|c|c|}
\hline Categoría & Variable observada & Sí & $\begin{array}{l}\text { Algunas } \\
\text { veces }\end{array}$ & No \\
\hline \multirow{3}{*}{$\begin{array}{l}\text { Comunicación entre } \\
\text { los padres }\end{array}$} & $\begin{array}{c}\text { Entre los padres: } \\
\text { constante y afectuosa }\end{array}$ & 1 & 4 & 3 \\
\hline & $\begin{array}{l}\text { Entre los padres y } \\
\text { el bebé: caricias y } \\
\text { conversación }\end{array}$ & 0 & 6 & 2 \\
\hline & $\begin{array}{c}\text { Con el personal de } \\
\text { salud }\end{array}$ & 2 & 2 & 4 \\
\hline \multirow[b]{2}{*}{ Estimulación táctil } & Uso de instrumentos & 0 & 1 & 7 \\
\hline & $\begin{array}{l}\text { Aplicación a través del } \\
\text { vientre materno }\end{array}$ & 0 & 4 & 4 \\
\hline \multirow{2}{*}{ Estimulación visual } & Luz natural $(n=7)$ & 4 & 1 & 2 \\
\hline & Luz artificial & 1 & 1 & 6 \\
\hline \multirow{3}{*}{ Estimulación auditiva } & Sonido & 0 & 3 & 5 \\
\hline & Habla & 0 & 4 & 4 \\
\hline & Música & 1 & 0 & 7 \\
\hline \multirow{3}{*}{ Estimulación motora } & Ejercicios físicos & 0 & 0 & 8 \\
\hline & Respiración & 0 & 0 & 8 \\
\hline & Equilibrio & 0 & 0 & 8 \\
\hline
\end{tabular}

Fuente: elaboración propia.

La información sobre los conocimientos, las experiencias y las prácticas de las madres obtenida en los grupos focales se relacionó con conocimientos generales. Aquellas manifestaron que la estimulación prenatal: "Ayuda a que sean más seguros de sí mismos, más amorosos e independientes [...] a desarrollar partes del cerebro, conexiones — se hacen más conexiones—, lo que permite que su cerebrito crezca mejor y sea más inteligente cuando nazca" (MGFl).

Reconocen que hay actividades que facilitan la estimulación, y refieren su experiencia: "Este tipo de estímulos se ha dado principalmente en actividades como bailar, hacer ejercicio y los desplazamientos horizontales y verticales (por ejemplo en un ascensor)" (MGF6).

Con el movimiento, la mayoría reportó dos sensaciones: una de fatiga y otra de relajación, y las relacionaron con la de bienestar: 
"Relajado, los músculos no están tensionados; el baile realmente es un ejercicio, entonces ayuda a relajar y a ejercitar no solo la mente, sino también el cuerpo, aunque se termina cansado" (MGF6).

A la pregunta sobre si los estímulos sensoriales, como las caricias, benefician al feto, las madres respondieron que los relacionaban con el afecto y la comunicación, además de verlos como un medio para detectar un mayor o un menor nivel de actividad de su bebé: "Es una manifestación de afecto, de ternura, de amor: cuando uno se identifica con otra persona y recibe de aquella esos sentimientos, uno se da cuenta de que la persona lo quiere. También es una forma de estimular el cariño, la parte sexual..." (MGF1). "Todo el día, eso es como un instintito, ¿no?, es como un instinto de uno estarse tocando el estómago" (MGF3).

En los grupos focales surgió el estímulo de temperatura. Las madres reportaron que los bebés "se esconden" cuando algún estimulo no es de su agrado. Por ejemplo, cuando realizaron las ecografías, todas refirieron que se volteaban y "pareciera que esquivaran" el transductor; algunas relacionaron este comportamiento con la temperatura del gel; otras relataron que sus bebés se "incomodan" si les cae agua fría en el vientre durante el baño: "Ya ellos saben quién es su mamá, su papá, cuando los está uno estimulando, tocándolos para alguna cosa. Cuando le ponen ese Doppler, a él no le gusta, entonces muchas veces le tiene uno que hablar para que se deje, porque él se esconde" (MGF3).

La estimulación visual la relacionaron con las respuestas ante la luz natural y artificial (con el celular):

Depende... Por ejemplo, para dormir, oscuro, y en la mañana no me gusta que esté con luz, que yo esté durmiendo no, no me gusta. Cuando estoy deprimida, no me gusta el sol, pero tampoco quedarme en la oscuridad. Por ejemplo, aquí que no bajan las persianas, no me gusta. Y para dormir, ojalá lo más oscuro que yo pueda estar... Yo le he puesto que lamparita, esa linternita, que el celular, y ellos reaccionan a esa luz... cuando les dan esos destellos así (la luz natural), por eso es que está en ese movimiento. (MGF5) [sic]

Las madres consideran transversal a todos los estímulos la estimulación auditiva: "Hablarle mucho, comentarle lo que uno haga, del papá, en compañía de música suave, de manera que el sonido no altere los nervios" (MGF2). Asimismo, hay que tener en cuenta la clase de música, porque puede afectar la personalidad del bebé: "Digamos que si uno le coloca reguetón o música así, fuerte, el bebé va a ser más extrovertido. También es importante el tipo de música que le gusta a la mamá, para que aquel sienta esa conexión (MGF2).

Una categoría emergente corresponde a la coherencia entre el estado emocional de la madre y el feto. En múltiples ocasiones, las madres relatan la relación entre los diferentes tipos de estímulos y cierta sensación que conduce a un estado emocional, y que, a su vez, guarda coherencia con el estado emocional de su bebé en formación, el cual logran identificar a partir de su actividad en el vientre: "Todo lo que siente uno es lo que él siente: cuando uno está contento, él está contento; cuando uno está triste, él también está triste. Todo eso sale así, así como uno lleve el embarazo, así mismito sale, ni más ni menos" (MGF4).

\section{Segunda fase: implementación de estrategias}

Al finalizar la primera fase, se identificaron vacíos en la información y necesidades educativas respecto a: 1) cómo se estimula al feto; 2) a partir de qué semana es viable el nacimiento del bebé; 3) cómo manejar al bebé una vez que nace y al estar en su hogar; 4) cuáles son las consecuencias de las enfermedades que padece la madre y cómo estas afectan la salud del bebé, y 5) cuántas son las semanas mínimas de estancia del bebé para que nazca sin complicaciones.

Lo anterior facilitó la planeación, concertación, diseño y ejecución de talleres educativos, así como la adecuación y orientación de las acciones terapéuticas en la atención prenatal. Se realizaron las actividades de intervención en coherencia con los resultados descritos, las necesidades de conocimiento y las prácticas identificadas. En aquellas se enseñó la estimulación prenatal, que reflejó cómo hacer la estimulación visual, táctil, vestibular y de movimiento. De fondo, se utilizó la música, como está descrito en la tabla 1.

El $40 \%$ de las madres participó en todos los talleres de estimulación prenatal y actividades terapéuticas; el $26 \%$ se vinculó a los talleres y a una de las actividades terapéuticas, y el $34 \%$ restante solo estuvo presente en los talleres de estimulación prenatal. Las participantes del estudio colaboraron y realizaron las actividades propuestas, incluyendo la elaboración del juguete con material desechable, según su gusto. En el transcurso de la actividad se consideró la condición de salud y la motivación de las madres. 


\section{Tercera fase: reflexión}

Al finalizar los talleres, las madres reconocieron los beneficios de la estimulación prenatal en el desarrollo del feto antes y después del parto. Comentaron acerca de las experiencias y de las sensaciones percibidas durante la aplicación de los estímulos, y ninguna manifestó aversión ni se presentó situación de emergencia alguna. Como datos relevantes de las actividades de estimulación, no se observaron limitaciones en el desplazamiento de un sitio a otro ni durante la ejecución de las actividades manipulativas.

Las coincidencias entre las respuestas de las madres ante las actividades permitió establecer que la comunicación abierta, interactiva, fluida con sus pares, los terapeutas y el personal de salud que las asistió en la sala fue inconforme. Al inicio, algunas madres, con lenguaje no verbal, se mostraron apáticas y poco expresivas durante la estimulación, pero al final se observó un cambio de actitud: una mayor empatía e interacción con objetos y estímulos.

Las madres construyeron elementos para estimular al bebé durante las actividades terapéuticas, y en medio de estas se observaron actitudes de apoyo entre las participantes e intercambios sobre sus emociones. El disfrute fue visible: la comunicación fue asertiva, fluida y coherente, así como la proactividad de las madres, que preguntaban y proponían alternativas frente a los aspectos de procedimiento; estuvieron atentas y concentradas, realizaron un trabajo colaborativo, los implementos los colocaban en su lugar, iniciaban y terminaban la actividad y tenían ritmo y secuenciación en su progreso.

Para medir el grado de satisfacción de las madres e indagar sobre las experiencias y percepciones de sus vivencias, se utilizaron las encuestas institucionales, y en particular los ítems que les concernían. Allí se identificó que todas las madres percibieron un trato adecuado, calificado con $5 / 5$, así como la información que recibieron, también calificada con $5 / 5$ en once ocasiones, excepto por dos de las gestantes, que puntuaron esta categoría con 4/5. Solo una de las madres calificó el ambiente físico, con un puntaje de $4 / 5$. Por otro lado, la pertinencia fue calificada con $5 / 5$ por trece madres, y respecto a la continuidad de la atención, cinco madres consideraron una puntuación de $5 / 5$, y dos gestantes la calificaron con 4/5.

\section{Discusión}

Los hallazgos del estudio pueden relacionarse con otras investigaciones desde tres aspectos fundamentales: 1) las características sociodemográficas y clínicas de las gestantes de alto riesgo; 2) la implementación de la estimulación prenatal y sus efectos en la ocupación de las madres, su sentido de autoeficacia y el desarrollo sensorial de los bebés, y 3) la relevancia del abordaje interdisciplinar en los procesos de estimulación prenatal.

Se identificó una relación entre la distribución de la población de las madres gestantes participantes y los hallazgos en la literatura. Mediante el $80 \%$ de los diagnósticos médicos de hospitalización se identificó preeclampsia, de forma coherente con la alta prevalencia de este trastorno en las gestantes $(3,7)$; de igual forma, la mitad de las madres presentaron una edad gestacional de 33 semanas, lo cual sugiere un alto riesgo obstétrico por debajo de las 37 semanas, y dicha morbilidad materna produce riesgo de prematurez y morbimortalidad neonatal $(8,10)$.

Las gestantes que participaron fueron expuestas a un aprendizaje o reaprendizaje de la estimulación prenatal. Compartieron conocimientos, experiencias, percepciones, prácticas y ocupaciones, lo cual permitió a los profesionales tener una visión general del proceso, así como comprender las subyacentes influencias articuladas en el compromiso ocupacional. Esto favoreció la creación de un espacio que les permitió establecer conexiones, tanto neurales, como afectivas, y en el cual primó la responsabilidad para entender la importancia de estimular al bebé que va a nacer (32-35).

Además, los ambientes hospitalarios afectan el acceso de una madre a las ocupaciones de la vida diaria, pues influyen en su eficacia y en la calidad del resultado, así como en la satisfacción de aquella respecto a su rendimiento. Cuando se adapta el ambiente, pueden lograrse cambios y transformaciones exitosas que favorecen el desempeño de las madres durante la hospitalización (33, 34). Las gestantes de este estudio participaron en la ejecución de las estrategias y fueron productivas en la construcción de un material para estimular a su bebé; se ocuparon en actividades con significado, por lo cual alcanzaron un estilo de vida equilibrado y funcional, y apreciaron cambios emocionales, facilitados por la creación de grupos de apoyo $(35,36)$.

Las ocupaciones compartidas facilitaron la ejecución, la comunicación fluida y el aprovechamiento del tiempo libre. Las 
actividades de estimulación prenatal y la preparación para la maternidad, con programas de abordaje colectivo, promueven la salud física y mental de la gestante, previenen complicaciones y, de esta forma, permiten centrarse en lo que les convoca la actividad; esto fue mencionado en los planteamientos de Verny (26), Aguilar (17), Prieto (19), Bello (28), Lobato (29), Federico (30) y Begoña (31). Además, se afirma que las ocupaciones se convierten en rutinas socialmente interactivas, y esta interacción social es recíproca y se arraiga en los compañeros de ocupaciones (36).

Por otro lado, la estimulación prenatal se ha estudiado principalmente desde el enfoque cuantitativo $(9,17-19)$, con aproximaciones teóricas que permiten observar la relevancia de esta práctica en la promoción de la salud (16). Esto contrasta con la metodología usada para este estudio, en el cual el enfoque cualitativo permitió comprender el fenómeno desde la perspectiva de las madres, y en consideración del significado otorgado a la ocupación y el desarrollo de autoeficacia y satisfacción personal, aspecto que releva la participación interdisciplinar con inclusión de los terapeutas ocupacionales en dichos procesos.

En consecuencia, los profesionales de la salud deben atender de forma interdisciplinar no solo las ocupaciones, sino también la variedad de factores que potencian y hacen que el compromiso y la participación de las madres promuevan aspectos positivos para su salud, desde donde pueden aportar $(35,37)$. Por esto, el apoyo terapéutico fue una guía para la estimulación, la interacción, el manejo de la experiencia del bebé y, como valor agregado, la concientización sobre la humanización de los profesionales en la prestación del servicio para las madres, quienes promovieron la integración permanente de actividades para esta población (37).

\section{Conclusiones}

Al participar en ocupaciones en el contexto hospitalario, las gestantes implementan nuevas formas de saber y hacer que les motiva a encontrar actividades significativas y de reaprendizaje de estimulación prenatal. Los terapeutas ocupacionales reconocen que para lograr una plena participación en la ocupación con significado y propósito, las gestantes deben realizar actividades que les permitan incorporar la capacidad, eficacia y goce en los procesos adquiridos y realizados.

Las herramientas conceptuales y de praxis del terapeuta ocupacional ofrecen calidad de vida a los seres ocupacionales. A las madres gestantes las apoyan para que ocupen el tiempo libre con actividades creativas, vean la vida de una forma diferente, aprendan otros modos de adquirir conocimiento y desarrollen su sensibilidad frente a los seres con los que interactúan.

Además, los abordajes grupales con implementación de estrategias de estimulación prenatal propician espacios que favorecen la creatividad, la regeneración de las fuerzas intelectuales, la construcción de identidad, la búsqueda de la felicidad y el sentido de pertenencia, lo cual permite que las gestantes, a partir de los conocimientos adquiridos, identifiquen sus capacidades de creación en la relación entre el campo del saber y su función social; se enseñen a relativizar su aplicación y a tender "puentes" entre el saber cultural y el saber cotidiano, entre la vida y el arte.

Finalmente, se observa que las ocupaciones promueven la salud física y mental de la gestante, con abordajes integrales e interdisciplinares, porque se centran en aprovechar el tiempo libre, al ejecutar actividades con propósito y modelar la estimulación prenatal.

Conflicto de intereses: ninguno declarado. 


\section{Referencias}

1. Royert J, Peñate M. Caracterización de las gestantes de alto riesgo obstétrico en el departamento de Sucre (Colombia), 2015. Salud Uninorte. 2016 sep.-dic.;32(3):452-60. Disponible en: http://rcientificas.uninorte.edu.co/index.php/salud/article/view/9137

2. Organización Mundial de la Salud. Mortalidad materna 2018 [Internet]. 2018 feb. [citado 2018]. Disponible en: http:// www.who.int/es/news-room/fact-sheets/detail/maternal-mortality

3. Organización Panamericana de la Salud. Protocolo de vigilancia epidemiológica de la mortalidad materna. Bogotá: s.e.; 2016.

4. Secretaría de Salud Pública Municipal de Cali. Análisis de la situación integrada de salud en el municipio de Santiago de Cali, periodo 2009 a 2016. Cali: s.e.; 2016.

5. Organización Mundial de la Salud. Estrategia mundial para la salud de la mujer, el niño y el adolescente (2016-2030). Ginebra: s.e.; 2015 .

6. Ministerio de Salud y Protección Social. Resolución 3202 de 2016 (25 de julio), por la cual se adopta el Manual Metodológico para la elaboración e implementación de las Rutas Integrales de Atención en Salud (RIAS), se adopta un grupo de Rutas Integrales de Atención en Salud desarrolladas por el Ministerio de Salud y Protección Social dentro de la Política de Atención Integral en Salud (PAIS) y se dictan otras disposiciones. Diario Oficial n. ${ }^{\circ} 49.947$.

7. Ministro de Salud y Protección Social y UNFPA. Protocolo de atención preconcepcional. Bogotá: Ministerio de Salud; 2014.

8. Donoso B, Oyarzún E. Embarazo de alto riesgo. Medwave. 2012 jun.;12(05): e5429 DOI: 10.5867/medwave.2012.05.5429

9. Morales S, García Y, Agurto M, Benavente A. La psicoprofilaxis obstétrica y la estimulación prenatal en diversas regiones del Perú. Horizonte Médico. 2014 ene.;14(1). 42-48. Disponible en: http://www.redalyc.org/articulo. oa?id=371637133008

10. Dalla Costa L, Cura C, Perondi A, França V, Bortoloti DS. Perfil epidemiológico de gestantes de alto risco. Cogitare Enfermagem. 2016 jun.;21(2):1-8. DOI: 10.5380/ce.v21i2.44192

11. Reynoso C, Crespo-Eguílaz N, Alcázar J, Narbona J. Motricidad fetal durante el segundo trimestre de gestación: Estudio ecográfico longitudinal. Anales de Pediatría. 2015 jul.;82(3):183-91. DOI: 10.1016/j.anpedi.2014.05.011

12. Guijarro-Villar C, Martínez-Lorca M, Zabala-Baños M. Conocimiento de la estimulación prenatal en los programas de educación maternal en atención primaria por parte de las mujeres gestantes. Rev Clin Med de Fam. 2016 feb.;9(2):8390. Disponible en: http://scielo.isciii.es/pdf/albacete/v9n2/original2.pdf

13. Organización Mundial de la Salud. Recomendaciones de la OMS sobre atención prenatal para una experiencia positiva del embarazo. Ginebra: Program HR; 2016. Disponible en: https://www.who.int/reproductivehealth/publications/maternal_perinatal_health/anc-positive-pregnancy-experience/es/

14. Lathrop B, Pritham U. A pilot study of prenatal care visits blended group and individual for women with low income. Nurs Womens Health. 2014 dic.;18(6):462-74. DOI: 10.1111/1751-486X.12159

15. Ashtarian H, Ghanbari M, Moradi A, Baigi L. Self-efficacy in Pregnant Women Referred to Health Centers in Delfan, Iran. Int J Health Life Sci. 2016 sep.;2(1):e74058. Disponible en: http://ijhls.com/en/articles/74058.html

16. Hurtado M, Cuadrado S, Herrán A. Hacia una pedagogía prenatal. Una propuesta educativa. Revista Iberoamericana de Educación. 2015 ene.;67(1):158-68. Disponible en: https://rieoei.org/RIE/article/view/268

17. Aguilar-Cordero M, Padilla-López, C. La estimulación prenatal: Resultados relevantes en el periparto. Nutrición Hospitalaria. 2015 nov.-dic.;27(6):2102-8. DOI: 10.3305/nh.2012.27.6.6019

18. García L, Flórez Y. Prácticas sobre estimulación prenatal que realizan las gestantes adultas asistentes a control prenatal en Sincelejo (Colombia). Salud Uninorte. 2008 ene.-jun.;12(4):31-39. Disponible en: http://rcientificas.uninorte.edu.co/ index.php/salud/article/viewArticle/1957 
19. Prieto-Bocanegra B. Prácticas de cuidado y su influencia durante la gestación en Tunja, Colombia. Revista Cubana de Enfermería. 2016 jul.-sep.;32(3):1-15. Disponible en: http://www.medigraphic.com/cgi-bin/new/resumen. cgi?IDARTICULO=68131

20. Zúñiga-González C, Jarquín-Saenz M, Martínez-Andrade E, Rivas-García J. Investigación acción participativa: un enfoque de generación del conocimiento. Revista Iberoamericana de Bioeconomía y Cambio Climático. 2016 abr.;2(1):218226. DOI: $10.5377 /$ ribcc.v2i1.5696

21. Vivar C, McQueen A, Whyte D, Canga-Armayor N. Primeros pasos en la investigación cualitativa: Desarrollo de una propuesta de investigación. Index de Enfermería. 2013 dic.;22:222-7.

22. Ministerio de Salud. Resolución 8430 de 1993 (4 de octubre), por la cual se establecen las normas científicas, técnicas y administrativas para la investigación en salud. Diario Oficial n. ${ }^{\circ} 49427$.

23. Hamui-Sutton A, Varela-Ruiz M. La técnica de grupos focales. Investigación en Educación Médica. 2013 ene.mar.;2(5):55-60. DOI: 10.1016/S2007-5057(13)72683-8.

24. Hernández-Sampieri R, Fernández-Collado C, Baptista-Lucio P. Metodología de la Investigación. 6. ${ }^{\text {a }}$ ed. México D. F.: McGraw-Hill; 2014. Cap. n. ${ }^{\circ} 14$, Los procesos mixtos de investigación; p. 453-459

25. Ludington S. How to have a smarter baby. Nueva York: Printing History; 1985.

26. Verny T. El vínculo afectivo con el niño que va a nacer. Barcelona: Urano; 1992.

27. Verny T. The secret life of the unborn child. Nueva York: Delta; 1988.

28. Bello M. Educación física fetal y posnatal. Revista Corporeizando. 2013 feb.;1(10):92-110. Disponible en: http://docplayer.es/55936682-Educacion-fisica-fetal-y-posnatal.html

29. Lobato-Rodríguez J. Respuesta fetal a la estimulación prenatal auditiva en gestantes del tercer trimestre de embarazo. Revista Peruana de Obstetricia y Enfermería. 2016 dic.;10(2):1-8.

30. Federico G. La música como organizadora desde el vientre materno [Internet]. 2015. [citado 2018 jun. 17]. Disponible en: http://gabrielfederico.com/papers/uruguay2Available

31. Begoña Ibarrola. Música para antes de nacer. Madrid: Ediciones SM (ISBN: 9788467552409); 2012.

32. Morozini M. Exploring the engagement of parents in the co-occupation of parent-child play: An occupational science's perspective. International Journal of Prevention and Treatment. 2015 abr.;4(2A):11-28.

33. American Occupational Therapy Association. Occupational therapy in the promotion of health and well-being. American Journal of Occupational Therapy. 2013 nov.;67:47-59. Disponible en: https://ajot.aota.org/article. aspx?articleid $=1853063$

34. Bar M, Jarus T. The effect of engagement in everyday occupations, role overload and social support on health and life satisfaction among mothers. International Journal of Environmental Research and Public Health. 2015;12(6):6045-65. DOI: $10.3390 /$ ijerph120606045

35. Kuo A. A transactional view: occupation as a means to create experiences that matter. Journal of Occupational Science. 2011 may.;18(2):131-8. DOI: 10.1080/14427591.2011.575759

36. Parra-Esquivel E. Análisis del concepto ‘justicia’ en terapia ocupacional. Revista de la Facultad de Medicina. 2015 jul.;63(3). DOI: 1015446/revfacmedv63n349629

37. Vidal-Martí A, Reyes-López M, Fernández-Rabasa Y, González-Cuesta G, Acosta-Garrido T, Yero-Arniella L. Preparación psicofísica para el parto: seguridad y satisfacción a las gestantes. MediSur. 2012 feb.;10(1):17-21. Disponible en: http://scielo.sld.cu/scielo.php?script=sci_arttext\&pid=S1727-897X2012000100003 